PROCEEDINGS OF THE

AMERICAN MATHEMATICAL SOCIETY

Volume 128, Number 4, Pages 1055-1062

S 0002-9939(99)05112-6

Article electronically published on July 28, 1999

\title{
FROM NÖRLUND MATRICES TO LAPLACE REPRESENTATIONS
}

\author{
GORD SINNAMON
}

(Communicated by Christopher D. Sogge)

\begin{abstract}
The existence of Laplace representations for functions in weighted Hardy spaces on the right half plane is established. The method uses an extension of an inequality involving Nörlund matrices and corresponding convolution operators on the line. Analogous inequalities are proved for power series representations of functions in weighted Hardy spaces on the disc.
\end{abstract}

\section{INTRODUCTION}

The map that associates an analytic function in the disc with its sequence of power series coefficients may be viewed as an extension of the Fourier transform on the circle. If the analytic function extends to be continuous on the circle, then the two coincide. The complex Laplace transform

$$
L F(z)=\int_{0}^{\infty} e^{-t z} F(t) d t, \quad \operatorname{Re} z>0,
$$

associates a (suitable) function defined on $(0, \infty)$ with an analytic function in the right half plane. This may be viewed as an extension of the Fourier transform on the line because if the analytic function extends to be continuous (and not too large) on the line $\operatorname{Re} z=0$, the Laplace transform $L F$ is just the (Poisson extension of the) Fourier transform of $F$. It is therefore appropriate to view the power series representation of an analytic function in the disc and the Laplace representation of an analytic function in the half plane as analogous constructions. Moreover, both are intimately related to the Fourier transform.

In this paper we explore the relationship between the weighted Hardy space norms of analytic functions in the disc and in the half plane and the weighted Lebesgue space norms of their power series and Laplace representations respectively. In particular we identify a large class of weighted Hardy spaces in the half plane whose functions do have Laplace representations.

Let $1 \leq p<\infty$ and suppose that $w(z)$ is a non-negative, locally integrable function defined on the disc $D=\{z:|z|<1\}$. The weighted Hardy space $H_{w}^{p}(D)$

Received by the editors May 18, 1998.

1991 Mathematics Subject Classification. Primary 42A38; Secondary 40 G05.

Key words and phrases. Nörlund matrix, Laplace representation, Fourier inequality, Hardy space, weight.

Support from the Natural Sciences and Engineering Research Council of Canada is gratefully acknowledged. 
is the collection of functions, analytic in $D$, which satisfy

$$
\|f\|_{H_{w}^{p}(D)}=\sup _{0<r<1}\left(\int_{-\pi}^{\pi}\left|f\left(r e^{i \theta}\right)\right|^{p} w\left(r e^{i \theta}\right) \frac{d \theta}{2 \pi}\right)^{1 / p}<\infty .
$$

Similarly, if the weight $w$ is defined in the right half plane $\{z: \operatorname{Re} z>0\}$, the weighted Hardy space $H_{w}^{p}$ is the collection of functions, analytic in the right half plane, satisfying

$$
\|f\|_{H_{w}^{p}}=\sup _{x>0}\left(\int_{-\infty}^{\infty}|f(x+i y)|^{p} w(x+i y) d y\right)^{1 / p}<\infty .
$$

The classical Hardy spaces $H^{p}(D)$ and $H^{p}$ are obtained by taking $w \equiv 1$.

It is well known that if $1<p \leq 2$, then every $f \in H^{p}(D)$ can be represented by a power series $\sum_{n=0}^{\infty} c_{n} z^{n}$ with $\left\{c_{n}\right\} \in l_{p^{\prime}}$ and almost as well known that every $f \in H^{p}$ can be represented as a Laplace transform $L F$ with $F \in L^{p^{\prime}}(0, \infty)$. (Here and throughout we use a prime ${ }^{\prime}$ to denote harmonic conjugate so that $1 / p+1 / p^{\prime}=1$.)

Laplace representations for functions in weighted Hardy spaces were introduced by Rooney in several papers leading up to [5]. He showed that if $1<r \leq \min \left(p, p^{\prime}\right)$, $p \leq q \leq r^{\prime}$, and $w(z)=|z|^{(p / r)-1}$, then every $f$ in $H_{w}^{p}$ has a representation $f=L F$ for some $F$ with $\int_{0}^{\infty}|F(t)|^{q} t^{\left(q / r^{\prime}\right)-1} d t<\infty$. The key to Rooney's approach was his proof of weighted Lebesgue norm inequalities for the Fourier transform. Benedetto, Heinig and Johnson, in [1] and [2], studied Fourier inequalities in weighted Lebesgue spaces and applied their results to give Laplace representations for functions in very general weighted Hardy spaces.

The results of the present paper do not rely on weighted Lebesgue space norm inequalities for the Fourier transform but are derived instead from inequalities for a convolution operator which corresponds, in sequence spaces, to the action of a Nörlund matrix. With this new approach we obtain Laplace representations for functions in weighted Hardy spaces for a class of weights that is not included in previous results. We include examples of power weights in this new class for which Laplace representations were not previously known.

Nörlund matrices are discussed in the next section together with a result illustrating their connection with convolution and power series representations. In Section 3, we extend the results for Nörlund matrices and prove the analogous convolution inequality on the half line. The final section contains the Laplace and power series representation theorems and examples.

\section{NÖRLUND MATRICES AND POWER SERIES}

Definition 2.1. Suppose that $\left\{a_{n}\right\}_{n>0}$ is a sequence of non-negative real numbers with $a_{0}>0$ and set $A_{n}=a_{0}+a_{1}+\cdots+a_{n}$. The Nörlund matrix $N_{a}$ is defined by $\left(N_{a}\right)_{n, k}=a_{n-k} / A_{n}$ for $0 \leq k \leq n$ and $\left(N_{a}\right)_{n, k}=0$ otherwise.

In 1984, Borwein and Cass showed that $N_{a}$ is a bounded operator on $l_{q}$ whenever $\left\{n a_{n} / A_{n}\right\}$ is a bounded sequence. We state this result as an inequality.

Proposition 2.2 (Borwein and Cass [3]). Suppose that $\left\{a_{n}\right\}_{n \geq 0}$ is a sequence of non-negative real numbers with $a_{0}>0$, define $A_{n}=a_{0}+\cdots+a_{n}$ as above, and suppose that $\left\{n a_{n} / A_{n}\right\}$ is bounded. Then, for $q \in(1, \infty)$, there exists a constant $C$ 
such that

$$
\left(\sum_{n=0}^{\infty}\left|\sum_{k=0}^{n} a_{n-k} x_{k}\right|^{q} A_{n}^{-q}\right)^{1 / q} \leq C\left(\sum_{k=0}^{\infty}\left|x_{k}\right|^{q}\right)^{1 / q}
$$

for all sequences $\left\{x_{k}\right\}_{k \geq 0}$.

We recognize the inner sum above as the coefficient of $z^{n}$ in the product of the two power series $\sum_{n=0}^{\infty} a_{n} z^{n}$ and $\sum_{n=0}^{\infty} x_{n} z^{n}$. Viewed in this way the inequality becomes a weighted convolution inequality with $\left\{A_{n}^{-q}\right\}$ as the weight sequence. To pass from the convolution inequality to our first theorem on power series representation we need two lemmas.

Lemma 2.3. (a) If $f$ is meromorphic in the unit disc $D$ and

$$
\sup _{0<r<1} \int_{-\pi}^{\pi}\left|f\left(r e^{i \theta}\right)\right|^{q} \frac{d \theta}{2 \pi}<\infty
$$

for some $q \geq 1$, then $f$ is analytic in $D$.

(b) If $f$ is meromorphic in the half plane $\{z: \operatorname{Re} z>0\}$ and

$$
\sup _{x>0} \int_{-\infty}^{\infty}|f(x+i y)|^{q} d y<\infty
$$

for some $q \geq 1$, then $f$ is analytic in $\{z: \operatorname{Re} z>0\}$.

Proof. It suffices to prove part (a) in the case $q=1$ since that imposes the weakest condition on $f$. First observe that the finiteness of the supremum in (a) as $r \rightarrow 0$ shows that $f$ is bounded near zero so $f$ has no pole at 0 . If we suppose that $f$ has a pole at a non-zero point $a \in D$, then an easy estimate shows that the above integral, with $r=|a|$, does not converge. We conclude that $f$ has no pole in $D$ and hence is analytic there. Although in part (b) the case $q=1$ is no longer a weaker condition on $f$, that observation is still true locally and a similar argument shows that the finiteness of the supremum in (b) precludes the possibility of $f$ having a pole in $\{z: \operatorname{Re} z>0\}$.

The next lemma shows that the hypothesis that $\left\{n a_{n} / A_{n}\right\}$ be bounded is enough to ensure that the $a_{n}$ are the power series coefficients of a function which is analytic in the disc. We thank David Borwein for the elegant proof.

Lemma 2.4. Let $a_{n}$ and $A_{n}$ be as in Proposition 2.2. Then the power series $\sum_{n=0}^{\infty} a_{n} z^{n}$ converges in the unit disc.

Proof. Choose a positive integer $M$ such that $n a_{n} \leq M A_{n}$. If we write this as $n\left(A_{n}-A_{n-1}\right) \leq M A_{n}$, it can be rearranged to become $A_{n} / A_{n-1} \leq n /(n-M)$. Now

$$
\frac{A_{n}}{A_{M}}=\prod_{k=M+1}^{n} \frac{A_{k}}{A_{k-1}} \leq \prod_{k=M+1}^{n} \frac{k}{k-M} \leq(1 / M !) n^{M}
$$

and we get $a_{n} \leq(M / n) A_{n} \leq(1 /(M-1) !) A_{M} n^{M-1}$ so $\limsup _{n \rightarrow \infty}\left|a_{n}\right|^{1 / n} \leq 1$ and the radius of convergence of the power series is at least 1 as required.

We restrict ourselves to the case $q=2$ in the next theorem to illustrate the method. A more general result may be found in Section 4 . 
Theorem 2.5. Let $a_{n}$ and $A_{n}$ be as in Proposition 2.2 and let $u(z)=\sum_{n=0}^{\infty} a_{n} z^{n}$. Then there exists a constant $C$ such that

$$
\left(\sum_{n=0}^{\infty}\left|b_{n}\right|^{2} A_{n}^{-2}\right)^{1 / 2} \leq C\|g\|_{H_{|u|-2}^{2}}
$$

for all analytic functions $g(z)=\sum_{n=0}^{\infty} b_{n} z^{n}$ in the unit disc.

Proof. First we note that, by Lemma 2.4, $u$ is analytic in the unit disc $D$. Fix $g(z)=\sum_{n=0}^{\infty} b_{n} z^{n}$, analytic in $D$. If $\|g\|_{H_{|u|-2}^{2}}$ is infinite, then there is nothing to prove so suppose it is finite. By Lemma 2.3(a), $f=g / u$ is analytic in $D$. Writing $f(z)=\sum_{n=0}^{\infty} x_{n} z^{n}$ we can express the coefficients of $g$ as

$$
b_{n}=\sum_{k=0}^{n} a_{n-k} x_{k} .
$$

Now we have, by Proposition 2.2 with $q=2$,

$$
\left(\sum_{n=0}^{\infty}\left|\sum_{k=0}^{n} a_{n-k} x_{k}\right|^{2} A_{n}^{-2}\right)^{1 / 2} \leq C\left(\sum_{k=0}^{\infty}\left|x_{k}\right|^{2}\right)^{1 / 2}
$$

which is, using the Parseval relation,

$$
\left(\sum_{n=0}^{\infty}\left|b_{n}\right|^{2} A_{n}^{-2}\right)^{1 / 2} \leq C\|f\|_{H^{2}}=C\|g\|_{H_{|u|-2}^{2}} .
$$

\section{Weighted CONVOLUtion InEQUALities}

Our object in this section is to formulate analogues of Proposition 2.2, in the first of which the sequence $\left\{a_{n}\right\}$ is replaced by a function on the half line. We consider two indices instead of one, allow $a$ to take complex values, and eliminate the condition that $\left\{n a_{n} / A_{n}\right\}$ be bounded. The expense of these changes is a more complicated definition of $A_{n}$ but this is offset, in Section 4 , by the benefit of being able to prove quite general extensions of Theorem 2.5.

We begin by proving a variant of Schur's lemma.

Lemma 3.1. Suppose that $X$ and $T$ are measure spaces, $1<p^{\prime} \leq q<\infty$ and $k(x, t)$ is a non-negative, measurable function on $X \times T$. Define $\gamma$ by $\gamma / q+\gamma / p=1$. If there exists a positive, measurable function $u$ on $T$ and a constant $C$ such that

$$
\int_{X} k(x, t)^{\gamma}\left(\int_{T} k(x, s)^{\gamma} u(s) d s\right)^{q / p} d x \leq C^{q} u(t)^{q / p}
$$

for almost every $t \in T$, then the operator

$$
K f(x)=\int_{T} k(x, t) f(t) d t
$$

is bounded from $L^{p^{\prime}}(T)$ to $L^{q}(X)$. Moreover, the norm of the operator $K$ does not exceed $C$. 
Proof. Let $f \in L^{p^{\prime}}(T)$. Since $u$ is positive, we can multiply and divide by $u^{1 / p}$ and apply Hölder's inequality with indices $p^{\prime}$ and $p$ to get

$$
\begin{aligned}
& \left(\int_{X}\left|\int_{T} k(x, t) f(t) d t\right|^{q} d x\right)^{1 / q} \\
\leq & \left(\int_{X}\left(\int_{T} k(x, t)^{\gamma / q+\gamma / p}|f(t)| u(t)^{-1 / p} u(t)^{1 / p} d t\right)^{q} d x\right)^{1 / q} \\
\leq & \left(\int_{X}\left(\int_{T} k(x, t)^{p^{\prime} \gamma / q}|f(t)|^{p^{\prime}} u(t)^{-p^{\prime} / p} d t\right)^{q / p^{\prime}}\left(\int_{T} k(x, s)^{\gamma} u(s) d s\right)^{q / p} d x\right)^{1 / q} .
\end{aligned}
$$

We continue the estimate by bringing the second inner integral inside the first and using Minkowski's integral inequality with index $q / p^{\prime}$. This yields

$$
\begin{aligned}
& \left(\int_{X}\left|\int_{T} k(x, t) f(t) d t\right|^{q} d x\right)^{1 / q} \\
\leq & \left(\int_{X}\left(\int_{T} k(x, t)^{p^{\prime} \gamma / q}|f(t)|^{p^{\prime}} u(t)^{-p^{\prime} / p}\left(\int_{T} k(x, s)^{\gamma} u(s) d s\right)^{p^{\prime} / p} d t\right)^{q / p^{\prime}} d x\right)^{1 / q} \\
\leq & \left(\int_{T}\left(\int_{X}\left[k(x, t)^{p^{\prime} \gamma / q}|f(t)|^{p^{\prime}} u(t)^{-p^{\prime} / p}\left(\int_{T} k(x, s)^{\gamma} u(s) d s\right)^{p^{\prime} / p}\right]^{q / p^{\prime}} d x\right)^{p^{\prime} / q} d t\right)^{1 / p^{\prime}} \\
= & \left(\int_{T}|f(t)|^{p^{\prime}} u(t)^{-p^{\prime} / p}\left(\int_{X} k(x, t)^{\gamma}\left(\int_{T} k(x, s)^{\gamma} u(s) d s\right)^{q / p} d x\right)^{p^{\prime} / q} d t\right)^{1 / p^{\prime}} \\
\leq & C\left(\int_{T}|f(t)|^{p^{\prime}} d t\right)^{1 / p^{\prime}}
\end{aligned}
$$

where the last inequality is just the hypothesis (3.1). This completes the proof.

Theorem 3.2. Suppose that $1<p^{\prime} \leq q<\infty$, a is a locally integrable, complexvalued function on $[0, \infty)$ and

$$
A(x)=\max \left(\left(\int_{0}^{x}|a(t)|^{\gamma} d t\right)^{1 / \gamma}, \sup _{0<t \leq x} t^{1 / \gamma}|a(t)|\right)
$$

where $1 / \gamma=1 / p+1 / q$. Then there exists a constant $C$, independent of a, such that

$$
\left(\int_{0}^{\infty}\left|\int_{0}^{x} a(x-t) F(t) d t\right|^{q} A(x)^{-q} d x\right)^{1 / q} \leq C\left(\int_{0}^{\infty}|F(t)|^{p^{\prime}} d t\right)^{1 / p^{\prime}}
$$

for all functions $F \in L^{p^{\prime}}(0, \infty)$.

Proof. Without loss of generality we may assume that $a$ is non-negative. By Lemma 3.1 with $k(x, t)=a(x-t) / A(x)$ and $u(t)=t^{-1 / q}$, it is enough to prove the estimates

$$
\int_{0}^{x}\left(\frac{a(x-s)}{A(x)}\right)^{\gamma} s^{-1 / q} d s \leq c_{1} x^{-1 / q} \text { and } \int_{t}^{\infty}\left(\frac{a(x-t)}{A(x)}\right)^{\gamma} x^{-1 / p} d x \leq c_{2} t^{-1 / p}
$$

for some constants $c_{1}$ and $c_{2}$ depending only on $p$ and $q$. 
Following the proof in [3], we look at the first estimate in two parts, splitting the range of integration at $s=x / 2$. If $0<s<x / 2$, then $0<x-s<x$ so the definition of $A$ yields $A(x) \geq(x-s)^{1 / \gamma} a(x-s)$ and we have

$$
\int_{0}^{x / 2}\left(\frac{a(x-s)}{A(x)}\right)^{\gamma} s^{-1 / q} d s \leq \int_{0}^{x / 2}(x-s)^{-1} s^{-1 / q} d s \leq q^{\prime}(x / 2)^{-1 / q}
$$

If $x / 2<s<x$, then

$$
\int_{x / 2}^{x}\left(\frac{a(x-s)}{A(x)}\right)^{\gamma} s^{-1 / q} d s \leq(x / 2)^{-1 / q} A(x)^{-\gamma} \int_{x / 2}^{x} a(x-s)^{\gamma} d s \leq(x / 2)^{-1 / q}
$$

To establish the second estimate we split the integral at $x=2 t$. Since $A(x) \geq$ $(x-t)^{1 / \gamma} a(x-t)$ we have

$$
\int_{2 t}^{\infty}\left(\frac{a(x-t)}{A(x)}\right)^{\gamma} x^{-1 / p} d x \leq \int_{2 t}^{\infty}(x-t)^{-1}(x-t)^{-1 / p} d x=p t^{-1 / p}
$$

The other part follows from the fact that $A$ is nondecreasing.

$$
\int_{t}^{2 t}\left(\frac{a(x-t)}{A(x)}\right)^{\gamma} x^{-1 / p} d x \leq t^{-1 / p} A(t)^{-\gamma} \int_{t}^{2 t} a(x-t)^{\gamma} d x \leq t^{-1 / p}
$$

This completes the proof.

Note that, for some functions $a$, we may have $A \equiv \infty$, in which case the theorem holds trivially.

Corollary 3.3. Suppose that $1<p^{\prime} \leq q<\infty, 1 / \gamma=1 / p+1 / q,\left\{a_{n}\right\}_{n \geq 0}$ is a sequence of complex numbers, and

$$
A_{n}=\max \left(\left(\sum_{k=0}^{n}\left|a_{k}\right|^{\gamma}\right)^{1 / \gamma}, \max _{0 \leq k \leq n} k^{1 / \gamma}\left|a_{k}\right|\right)
$$

Then there exists a constant $C$, independent of the sequence $\left\{a_{n}\right\}$, such that

$$
\left(\sum_{n=0}^{\infty}\left|\sum_{k=0}^{n} a_{n-k} x_{k}\right|^{q} A_{n}^{-q}\right)^{1 / q} \leq C\left(\sum_{k=0}^{\infty}\left|x_{k}\right|^{p^{\prime}}\right)^{1 / p^{\prime}}
$$

for all complex sequences $\left\{x_{k}\right\}_{k \geq 0}$.

This discrete version is proved in just the same way as Theorem 3.2. It does generalize Proposition 2.2 since if $p^{\prime}=q,\left\{a_{n}\right\}$ is a non-negative sequence, and $\left\{n a_{n} /\left(\sum_{k=0}^{n} a_{k}\right)\right\}$ is bounded, then $\max _{0 \leq k \leq n} k a_{k}$ is bounded above by a constant multiple of $\sum_{k=0}^{n} a_{k}$ and we see that the new definition of $A_{n}$ is comparable to the original one. However, because of the new definition of $A_{n}$ it is not, in general, a theorem about Nörlund matrices.

\section{LAPlace REPRESENTATions IN Weighted $H^{p}$}

Theorem 2.5 began with a sequence $\left\{a_{n}\right\}$ and defined $u(z)=\sum_{n=0}^{\infty} a_{n} z^{n}$ to get the Hardy space $H_{|u|^{-2}}^{2}(D)$. Using this as a model we will start with a function $a$ and define $u$ to be the Laplace transform

$$
u(z)=\int_{0}^{\infty} e^{-z t} a(t) d t
$$


This entails placing a condition on the function $a$, namely that the integral in (4.1) converges absolutely to an analytic function on $\{z: \operatorname{Re} z>0\}$. This is a relatively mild condition on $a$, weaker than $a \in L^{1}(0, \infty)+L^{\infty}(0, \infty)$ but stronger than the local integrability assumed in Theorem 3.2.

Theorem 4.1. Suppose that $1<p \leq p^{\prime} \leq q<\infty$ and $a$ is a locally integrable, complex-valued function such that the integral in (4.1) converges absolutely to an analytic function $u$ on $\{z: \operatorname{Re} z>0\}$. Then to every $g \in H_{|u|-p}^{p}$ there corresponds a function $G \in L_{A^{-q}}^{q}(0, \infty)$ such that $g=L G$ and

$$
\left(\int_{0}^{\infty}|G|^{q} A^{-q}\right)^{1 / q} \leq C\|g\|_{H_{|u|-p}^{p}} .
$$

Here $A$ is defined by (3.2) and $C$ is a constant independent of $a$ and $g$.

Note that if $A \equiv \infty$ the representing function $G$ still exists but the norm inequality is trivial.

Proof. Suppose that $g \in H_{|u|^{-p}}^{p}$ and define $f=g / u$. By Lemma 2.3(b), $f$ is analytic and hence $f \in H^{p}$. Since $1<p \leq p^{\prime}$, we may use the special case $p=r=q^{\prime}$ of [5. Theorem 4.2] (originally due to Doetsch [4]) to see that $f$ is the Laplace transform $L F$ of some function $F \in L^{p^{\prime}}(0, \infty)$ and that

$$
\left(\int_{0}^{\infty}|F|^{p^{\prime}}\right)^{1 / p^{\prime}} \leq M\|f\|_{H^{p}}
$$

for some constant $M$ independent of $f$.

For each $z$ with $\operatorname{Re} z>0$, since

$$
\int_{0}^{\infty}\left|e^{-z t} F(t)\right| d t \leq\left(\int_{0}^{\infty} e^{-p(\operatorname{Re} z) t} d t\right)^{1 / p}\left(\int_{0}^{\infty}|F(t)|^{p^{\prime}} d t\right)^{1 / p^{\prime}}<\infty
$$

and since

$$
\int_{0}^{\infty}\left|e^{-z x} a(x)\right| d x
$$

is finite by hypothesis, we see that $e^{-z t} F(t) e^{-z(x-t)} a(x-t) \chi_{(0, x)}(t)$ is integrable on $(0, \infty) \times(0, \infty)$. By Fubini's theorem,

$$
G(x)=\int_{0}^{x} a(x-t) F(t) d t
$$

is defined for almost every $x>0$ and $e^{-z x} G(x)$ is integrable on $(0, \infty)$. Moreover, interchanging the order of the iterated integrals yields

$$
\int_{0}^{\infty} e^{-z x} G(x) d x=\int_{0}^{\infty} e^{-z t} F(t) \int_{t}^{\infty} e^{-z(x-t)} a(x-t) d x d t=f(z) u(z)=g(z)
$$

and we conclude that $g=L G$.

By Theorem 3.2 we have, using (4.2),

$$
\left(\int_{0}^{\infty}|G|^{q} A^{-q}\right)^{1 / q} \leq C\left(\int_{0}^{\infty}|F|^{p^{\prime}}\right)^{1 / p^{\prime}} \leq C M\|f\|_{H^{p}}=C M\|g\|_{H_{|u|}^{p}-p}
$$

so $G \in L_{A^{-q}}^{q}(0, \infty)$ as required. 
Example 4.2. Suppose that $1<p \leq p^{\prime} \leq q<\infty$. Take $a(t) \equiv 1$ and check that (3.2) and (4.1) yield $A(x)=x^{1 / q+1 / p}$ and $u(z)=1 / z$ respectively. Theorem 4.1 shows that every function $g \in H_{|z|^{p}}^{p}$ has a Laplace representation $g=L G$ where the function $G$ satisfies the inequality

$$
\|G\|_{L_{t^{-1-q / p}}^{q}(0, \infty)} \leq C\|g\|_{H_{\left.|z|\right|^{p}}^{p}} .
$$

These power weights are not included in Rooney's results or in those of Benedetto, Heinig and Johnson. This is explained by the reliance of those papers on Lebesgue space inequalities: The Lebesgue space Fourier inequality that corresponds to (4.3) does not hold. This negative result follows from [1, Theorem 2] because the weight pair $\left(t^{-1 / q-1 / p}, t\right)$ does not satisfy the $F_{p, q}$ condition defined there.

Example 4.3. Suppose that $1<p \leq p^{\prime} \leq q<\infty$. Fix real numbers $\alpha$ and $r$ with $0<r<q^{\prime}$. Set $a(t)=e^{i \alpha t} t^{1 / r}-1 / p-1$. Straightforward calculations yield $A(x) \approx t^{-1+1 / r+1 / q}$ and $u(z) \approx(z-i \alpha)^{1 / p-1 / r}$. Theorem 4.1 shows that every function $g \in H_{|z-i \alpha|^{p / r-1}}^{p}$ has a Laplace representation $g=L G$ for some function $G \in L_{t^{q-q / r-1}}^{q}(0, \infty)$.

The analogue of Theorem 4.1 in the disc is not needed to prove the existence of power series representations, which exist for all functions analytic in $D$, but the result is still of interest because the coefficient sequence is shown to be in a weighted $l_{q}$ space.

Theorem 4.2. Suppose that $1<p \leq p^{\prime} \leq q<\infty$ and $\left\{a_{n}\right\}_{n \geq 0}$ is a sequence of complex numbers satisfying lim $\sup _{n \rightarrow \infty}\left|a_{n}\right|^{1 / n} \leq 1$, and set $u(z)=\sum_{n=0}^{\infty} a_{n} z^{n}$. If $g(z)=\sum_{n=0}^{\infty} b_{n} z^{n}$ is analytic in the unit disc, then

$$
\left(\sum_{n=0}^{\infty}\left|b_{n}\right|^{q} A_{n}^{-q}\right)^{1 / q} \leq C\|g\|_{H_{|u|-p}^{p}(D)} .
$$

Here $A_{n}$ is defined by (3.3) and $C$ is a constant independent of $\left\{a_{n}\right\}$ and $g$.

The proof is a simple combination of the techniques used in Theorem 2.5 and Theorem 4.1 and is therefore omitted.

\section{REFERENCES}

1. J. J. Benedetto and H. P. Heinig, Weighted Hardy spaces and the Laplace transform, Harmonic Analysis Conference, Cortona Italy. Lecture Notes in Mathematics, vol. 992, Springer-Verlag, Berlin, 1982, pp. 240-277. MR 85j:44001

2. J. J. Benedetto, H. P. Heinig and R. Johnson, Weighted Hardy spaces and the Laplace transform II, Math. Nachr. 132 (1987), 29-55. MR 88m:44001

3. F. P. Cass and D. Borwein, Nörlund matrices as bounded operators on $l_{p}$, Arch. Math. 42 (1984), 464-469. MR 85j:40020

4. G. Doetsch, Bedingungen für die Darstellbarkeit einer Funktion als Laplace-Integral und eine Umkehrformel für die Laplace-Transformation, Math. Z. 42 (1937), 263-286.

5. P. G. Rooney, Generalized $H_{p}$ spaces and Laplace transforms, Abstract Spaces and Approximation, Proceedings of the Conference held at the Mathematical Research Institute at Oberwolfach, Black Forest, July 18-27, 1968 (P. L. Butzer and B. Sz-Nagy, ed.), Birkhauser Verlag, Basel, 1969, pp. 258-269. MR. 54:556

Department of Mathematics, University of Western Ontario, London, Ontario, Canada N6A 5B7

E-mail address: sinnamon@uwo.ca 